\title{
Use of E-Learning to Advance Nursing Education in Rwanda
}

\author{
Mary Murebwayire ${ }^{1}$, Innocent Biroli ${ }^{2}$, Helen Ewing ${ }^{2}$ \\ ${ }^{1}$ Ministry of Health, Rwanda; \\ ${ }^{2}$ University of Rwanda, College of Medicine and Health Sciences, Rwanda
}

\section{Background}

Most nursing care in Rwanda is provided by high school trained nurses. As of 2011, there were 6,970 nurses in Rwanda with $90 \%$ trained at the high school level. The Ministry of Health has set the goal of upgrading high school trained nurses to a diploma by 2020 . To assist in educating nurses and advancing their education, E-Learning (distance education) has been adopted as a model to support this education initiative.

\section{Description}

This is a description of the E-Learning Advanced Nursing Diploma program which was launched in 2012 to educate nurses trained at a high school level, to a nursing diploma. The goal was to develop a more skilled and educated nursing workforce. Electronic learning (E-Learning) uses the internet as a medium for interaction between students and teachers. The curriculum model used has $40 \%$ of the curriculum delivered in a face to face environment, and 60\% delivered through self-directed learning within the Moodle Learning Management System. As much of the Rwandan population (80\%) lives in rural areas, distance education can allow many nurses to obtain further education that was not available in the past. This increased education is critical to increase the competency levels of nurses and consequently the quality of the healthcare delivered.

\section{Lessons learned}

Over 800 nurses have commenced the program. There have been many challenges to implementing e-learning. These include a) Unstable Learning Management System, b) Inconsistent internet connectivity, c) Varying levels of informational technology literacy d) Language difficulties, and e) Altering faculty workloads and responsibilities with the new mode of education.

\section{Conclusions}

As it is early in the implementation of this innovative project, outcomes are limited. The first cohort of students (298) have completed the program and are preparing to write their licensure examinations. Program evaluation is a priority for the next year to determine the strengths of the program and areas for improvement.

Key words: e-learning, distance education, online learning, nursing, curriculum 